\section{P-60 GROWING OUR EVALUATION EXPERTISE}

${ }^{1}$ Jane Berg, ${ }^{1}$ Liz Reed, ${ }^{2}$ Ann Ooms. ${ }^{1}$ Princess Alice Hospice, Esher, UK; ${ }^{2}$ Kingston University/ St George's, University of London, UK

10.1136/bmjspcare-2018-hospiceabs.85

Background As a hospice we are nimble in our responses to the local population, developing service innovations and initiating new projects in response to identified needs. In 2017 we recognised that we are not always consistent in evaluating the benefits and challenges of these projects. We want to ensure that service developments are evaluated for cost effectiveness and benefit to our patient population. Our evaluation expertise was based in the research team and a limited resource.

Aim To expand our capacity and expertise in conducting evaluations and to embed this in a variety of departments.

Method Having secured funding from the Health Innovation Network (HIN) we are developing a team of Hospice Evaluation Champions (HECs) drawn from clinical and non- clinical staff. Guided by an external expert, the team meet on a regular basis to work on individual projects related to their own departments/projects and to learn together about the techniques and methodology appropriate to conducting a meaningful evaluation.

Results The programme of monthly workshops, individual supervision and peer support has resulted in the following ongoing evaluation projects

- Referrals to the medical team (Medical Consultant)

- Discharge Buddy introduction (Volunteer Manager)

- Introduction of IPOS (Quality Improvement Manager)

- Enhanced Support Service (Nurse Consultant)

- Introduction of HECs (Research Lead)

In conducting the evaluations the HECs have developed understanding and experience in using a range of tools including focus groups, interviews, questionnaires, diaries, and interrogation of existing data. They have also been supported in reporting and dissemination of evaluation results.

Dissemination On completion of the one year HEC project(s) the results will be disseminated through the hospice networks, through the HIN networks and through publication in journal articles. HECs will provide a cluster of expertise which will be able to support both internal and partnership projects in the future.

\section{P-61 HOW ARE YOU? THE USE OF IPOS IN CLINICAL ASSESSMENTS}

Nigel Dodds. St Christopher's Hospice, London, UK

10.1136/bmjspcare-2018-hospiceabs.86

St Christopher's Hospice teams have now worked with the Outcome Assessment Complexity Collaborative suite of measures for a number of years. The data they generate provides a valuable tool to support clinical decision making and care. From these experiences, we have learned the IPOS tool can be used to structure and lead a patient-centred holistic assessment. Therefore, with some minor adjustments, to include questions relating to patient wishes and goals, along with a patient information leaflet, to make the tool more 'patient friendly', we now have a variation of the IPOS tool to structure all of our formal clinical assessments in our different care settings.

This change supports our junior clinical staff to develop skills and competence in undertaking a palliative care holistic assessment; it also helps to standardise each patient assessment; furthermore, it enables clinicians to evaluate the impact of the previous assessment against the current one. The shift in practice has begun to provide large quantities of data, which improves our understanding of phase related symptom burden.

In this presentation, there will be a focus on describing the journey we have been on, in relation to adjusting the IPOS tool, and expanding the use of the IPOS tool; and also a focus on assessment items our teams feel they struggle with, alongside data which reflects the experience of patient assessment using the St Christopher's assessment tool.

\section{P-62 COMPARISON OF FAMILY AND STAFF MEMBERS' ASSESSMENTS OF HOSPICE INPATIENTS' SYMPTOMS USING IPOS}

${ }^{1} \mathrm{H}$ Lucy Thomas, ${ }^{1}$ Sharon Clazie, ${ }^{2}$ Andre Charlett, ${ }^{1}$ Pia Amsler. ${ }^{1}$ Isabel Hospice, Welmyn Garden City, UK; ${ }^{2}$ Public Health England, London, UK

\subsection{6/bmjspcare-2018-hospiceabs.87}

Background The Integrated Palliative Care Outcome Scale (IPOS) is a widely used questionnaire of patients' palliative care needs. If a patient is too unwell to complete an IPOS, a proxy respondent (family or staff) completes one on their behalf. Previous studies have compared proxy respondents' assessments of patient symptoms using a variety of scales, but none have used IPOS. There is therefore a lack of evidence to guide the selection of the most appropriate proxy IPOS respondent.

Aims To measure the agreement between patients and family members, doctors, nurses and nursing assistants (NSA) in assessing patients' concerns using IPOS.

Methods Once a week over one year, for each patient in our inpatient unit who had completed their own IPOS, a family member, doctor, nurse and NSA completed a proxy IPOS. We measured agreement using weighted Kappa statistics.

Results We had 95 response sets (patient plus at least one staff or family). Proxy respondents' agreement with patients was only moderate or fair for each IPOS item (Kappa 0.2-0.6). Overall, family members agreed most closely with patients (Kappa 0.58). There was no difference in agreement between different staff groups. For the majority of IPOS items, staff gave significantly lower ratings than patients. For a small number of items, family gave significantly higher ratings than patients. Both staff and family significantly over-estimate the patient not feeling at peace.

Conclusions Whenever possible, patients should be supported to complete their own IPOS, as proxy respondents do not agree strongly with patients. When patients are unable to complete an IPOS, family members are the most appropriate proxy. Under-estimation of issues by staff has implications for our ability to accurately assess and address our patients' needs. Conversely, over-estimation of issues, particularly the extent to which a patient feels at peace, may lead to under-estimation of quality of life. 